This is an author produced version of a paper published in System Modeling and Optimization. This paper has been peer-reviewed but does not include the final publisher proof-corrections or journal pagination.

Citation for the published paper:

Baravdish, George; Cheng, Yuanji; Svensson, Olof; Åström, Freddie. (2016). Extension of $p$-Laplace operator for image denoising. System Modeling and Optimization, p. null

URL: https://doi.org/10.1007/978-3-319-55795-3_9

Publisher: Springer

This document has been downloaded from MUEP (https://muep.mah.se) / DIVA (https://mau.diva-portal.org). 


\title{
Extension of $p$-Laplace Operator for Image Denoising
}

\author{
George Baravdish ${ }^{1}$, Yuanji Cheng ${ }^{2}$, Olof Svensson ${ }^{1}$, and Freddie Åström ${ }^{3 \star}$ \\ 1 Linköping University, Sweden \\ \{george.baravdish, olof.svensson\}@liu.se \\ 2 Malmö University, Sweden \\ yuanji.cheng@mah.se \\ ${ }^{3}$ Heidelberg Collaboratory for Image Processing, Heidelberg University, Germany \\ freddie.astroem@iwr.uni-heidelberg.de
}

\begin{abstract}
We introduce a novel operator $\Delta_{(p, q)}$ as an extended family of operators that generalize the $p$-Laplace operator. The operator is derived with an emphasis on image processing applications, particularly with a focus on image denoising applications. We propose a non-linear transition function, coupling $p$ and $q$, which yields a non-linear filtering scheme analogous to adaptive spatially dependent total variation and linear filtering. Well-posedness of the final parabolic PDE is established via perturbation theory and connection to classical results in functional analysis. Numerical results demonstrates the applicability of the novel operator $\Delta_{(p, q)}$.
\end{abstract}

Keywords: $p$-Laplace operator, parabolic equations, image denoising, anisotropic diffusion, inverse problems.

\section{Introduction}

A well known inverse problem in image processing is image denoising [4]. In the last decades the energy functional approach together with its corresponding Euler-Lagrange (E-L) equation has attracted great attention in solving inverse problems applied to image reconstruction. One important case of E-L equations is the one which involves the $p$-Laplace operator:

$$
\Delta_{p} u=\operatorname{div}\left(|\nabla u|^{p-2} \nabla u\right), \quad p \geq 1,
$$

associated with the evolution equation of $p$-Laplacian:

$$
\left\{\begin{aligned}
\partial_{t} u-\Delta_{p} u & =0, \text { in } \Omega \times(0, T) \\
u(0) & =u_{0}, \text { in } \Omega \\
\partial_{n} u & =0, \text { on } \partial \Omega \times(0, T)
\end{aligned}\right.
$$

\footnotetext{
* Acknowledgment Support by the German Science Foundation and the Research Training Group (GRK 1653) is gratefully acknowledged.
} 
where $\Omega$ is a bounded domain in $\mathbf{R}^{2}$ and $u_{0}: \Omega \rightarrow \mathbf{R}$ is a given degraded image [5], [8], [14] and $\nabla u$ is the gradient. The degenerate parabolic equation in (2) has been studied by many authors and we limit ourselves here to refer the reader to [11]. It is well known that the case $p=2$ will give the linear Gaussian filter, which however, impose strong spatial regularity and therefore image details such as lines and edges are oversmoothed. The case $p=1$ is often refereed to as the method of total variation [14] while $p=0$ is an instance of the so called balanced forward backward evolution [10].

In this work we studied a decoupled form of the $p$-Laplace operator, expressed as a non-linear combination of the $\Delta_{1}$ and $\Delta_{\infty}$ operators. We call our new operator $\Delta_{(p, q)}$. Using established existence theory we show that the corresponding perturbed parabolic equation is well-posed and close to the original operator.

In section 2 we review some of the properties of the $p$-Laplace operator applied in image denoising and compare it with Perona-Malik models. Our main contribution is in section 3 where we extend the $p$-Laplace operator to a new operator $\Delta_{(p, q)}$ with focus on image denoising. The corresponding variable version $\Delta_{(p(x), q(x))}$ is considered in section 4 . Finally, the applicability of $\Delta_{(p, q)}$ is demonstrates by numerical results in section 5 .

\section{$2 \quad p$-Laplacian for Image Denosing}

An important feature in any evolution process for image denoising is the preservation of certain geometrical features of the underlying image. In the case of image restoration these features include edges and corners. It is straight-forward to express the $p$-Laplace operator (1) as:

$$
\Delta_{p} u=|\nabla u|^{p-1} \Delta_{1} u+(p-1)|\nabla u|^{p-2} \Delta_{\infty} u,
$$

where $\Delta_{1} u=\operatorname{div}\left(\frac{\nabla u}{|\nabla u|}\right), \Delta_{\infty} u=\frac{\nabla u}{|\nabla u|} \cdot\left(D^{2} u\right) \frac{\nabla u}{|\nabla u|}$ and $D^{2} u$ is the Hessian of $u$. However, an intuitive way to represent $\Delta_{p}$, giving direct interpretation of the diffusivity directions is to express $\Delta_{p}$ by using Gauge coordinates $(x, y) \rightarrow(\eta, \xi)$ :

$$
\Delta_{p} u=|\nabla u|^{p-2}\left(u_{\xi \xi}+(p-1) u_{\eta \eta}\right)
$$

where

$$
\eta=\frac{\nabla u}{|\nabla u|}, \quad \xi=\frac{\nabla^{\perp} u}{|\nabla u|}
$$

and

$$
u_{\xi \xi}=|\nabla u| \Delta_{1} u, \quad u_{\eta \eta}=\Delta_{\infty} u .
$$

From (4) it is now clear that $\Delta_{p}$ imposes the same diffusivity strength in both directions $\xi$ and $\eta$ independent of the magnitude of the gradient. In an attempt to resolve this drawback, Perona and Malik [13] proposed to replace $|\nabla u|^{p-2}$ with $g\left(|\nabla u|^{2}\right)$ in (1). The idea is the weight should satisfy $g(s) \rightarrow 0, s \rightarrow \infty$ and $g(s) \rightarrow 1, s \rightarrow 0^{+}$. Perona and Malik studied weights like $g=k /(k+s)$ 


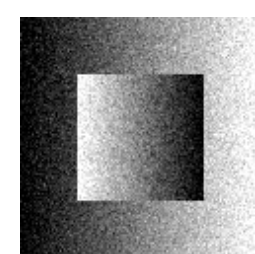

Noisy

PSNR: 22.2

SSIM: 0.30

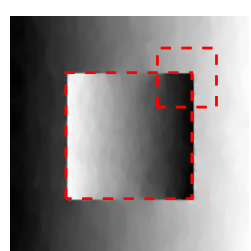

$\Delta_{(p(x), q(x))}$

0.96

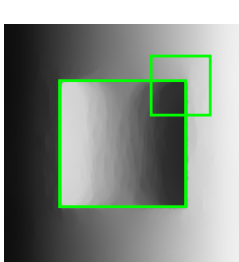

TV

28.3

0.94

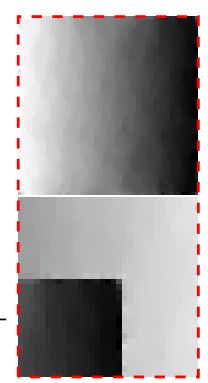

$\Delta(p(x), q(x))$

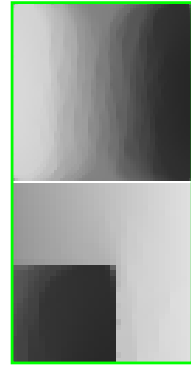

TV

Fig. 1: Synthetic test image with 20 standard deviations of noise (left) and the obtained results for TV (green/thick) and the $\Delta_{(p(x), q(x))}$-operator (red/dashed). Both error measures improve with our operator and shows less staircasing artifacts while preserving corner points and edges as presented in the detailed images.

and $g=e^{-k s}$ and demonstrated the advantages of these weight functions for edge preservations. Rewriting the operator given by the P-M method in Gauge coordinates, we obtain

$$
\operatorname{div}\left(g\left(|\nabla u|^{2}\right) \nabla u\right)=g\left(|\nabla u|^{2}\right) u_{\xi \xi}+\phi\left(|\nabla u|^{2}\right) u_{\eta \eta}
$$

where $\phi(s)=(s g(s))^{\prime}$. Thus the diffusion in the direction $\eta$ differs from the diffusion in the direction $\xi$. Since $\phi(s)$ is negative for large $s$, the evolution will be of backward diffusion effect near edges. The backward evolution cause problem for the well-posedness of the model and could also lead to staircasing problem [18].

The Perona-Malik PDE is a forward-backward type equation, the diffusion is forward in the region $\{|\nabla u|<k\}$ while it is backward and hence ill-posed in the region $\{|\nabla u|>k\}$. From evolution point of view, the forward-backward type equation may have infinitely many solutions. From the variational minimization perspective the energy functional may have infinitely many minima [18]. To overcome the ill-posedness in the Perona-Malik model, the authors in [18] introduced a regularization and proposed the following model

$$
\left.\partial_{t} u=\operatorname{div}\left(g\left(\left|\nabla G_{\sigma} * u\right|\right) \nabla u\right)\right),
$$

where $G_{\sigma}$ is a Gaussian function with standard deviation $\sigma$. A similar but time dependent variance $\sigma(t)$ was used in [17]. However, it's not always straight forward how to choose $\sigma(t)$ since it should neither decay too fast nor too slow during the evolution. 


\section{$3 \quad$ Extending the $p$-Laplace Operator}

\subsection{Anisotropic decomposition via constant coefficients}

The $p$-Laplace operator in (1), is an isotropic operator and expanding the divergence we obtain the equivalent form

$$
\Delta_{p} u=\partial_{x}\left(\left[\left(\partial_{x} u\right)^{2}+\left(\partial_{y} u\right)^{2}\right]^{\frac{p-2}{2}} \partial_{x} u\right)+\partial_{y}\left(\left[\left(\partial_{x} u\right)^{2}+\left(\partial_{y} u\right)^{2}\right]^{\frac{p-2}{2}} \partial_{y} u\right) .
$$

An anisotropic behavior of the $\Delta_{p}$-operator is induced by suppressing mixed derivatives, i.e., we define

$$
L_{(p, p)} u=\partial_{x}\left(\left|\partial_{x} u\right|^{p-2} \partial_{x} u\right)+\partial_{y}\left(\left|\partial_{y} u\right|^{p-2} \partial_{y} u\right), \quad 1 \leq p<\infty .
$$

In the case $p=1,(8)$ is known as isotropic TV and (9) is anisotropic TV [9]. By decoupling the exponents in (9) one obtains the operator $L_{\left(p_{1}, p_{2}\right)}$

$$
L_{\left(p_{1}, p_{2}\right)} u=\partial_{x}\left(\left|\partial_{x} u\right|^{p_{1}-2} \partial_{x} u\right)+\partial_{y}\left(\left|\partial_{y} u\right|^{p_{2}-2} \partial_{y} u\right), \quad 1 \leq p_{1}, p_{2}<\infty .
$$

which has previously appeared in fluid mechanics and we refer to [2], [3].

Next, to see how the diffusion appears in the operator (10) we reformulate it in Gauge coordinates (5) by making the following definition.

Definition 1. The $L_{\left(p_{1}, p_{2}\right)}$-operator is given by

$$
L_{\left(p_{1}, p_{2}\right)} u=\partial_{\xi}\left(\left|\partial_{\xi} u\right|^{p_{1}-2} \partial_{\xi} u\right)+\partial_{\eta}\left(\left|\partial_{\eta} u\right|^{p_{2}-2} \partial_{\eta} u\right),
$$

where $1 \leq p_{1}, p_{2}<\infty$.

The above operator $L_{\left(p_{1}, p_{2}\right)}$ is in fact a generalization of several known operators. We have

1. The case $p_{1}=p_{2}=2$. The operator $L_{(2,2)}$ in (11) is the Laplacian, by now well studied. Due to the Laplacian's rotation invariance property we get

$$
L_{(2,2)} u=\partial_{\xi}\left(\partial_{\xi} u\right)+\partial_{\eta}\left(\partial_{\eta} u\right)=\Delta u=u_{x x}+u_{y y} .
$$

2. The case $p_{1}=2$ and $p_{2}=1$. The operator $L_{\left(p_{1}, p_{2}\right)}$ in (11) is then given by

$$
L_{(2,1)} u=\partial_{\xi}\left(\partial_{\xi} u\right)+\partial_{\eta}\left(\left|\partial_{\eta} u\right|^{-1} \partial_{\eta} u\right) .
$$

Since we have $\left|\partial_{\eta} u\right|^{-1} \partial_{\eta} u=1$, it follows that $L_{(2,1)} u=u_{\xi \xi}$. In Cartesian coordinates this corresponds to

$$
L_{(2,1)} u=|\nabla u| \Delta_{1} u
$$

i.e. the mean curvature equation (see e.g., [7]). The corresponding regularized (weighted) mean curvature equation is given by

$$
\partial_{t} u=g\left(\left|\nabla G_{\sigma} * u\right|\right)|\nabla u| \Delta_{1} u
$$

previously studied in the context of image analysis, see e.g., [1]. 
3. The case $p_{1}=2$ and $p_{2}=p \in(1,2)$. It follows from (11) that

$$
\begin{aligned}
L_{(2, p)} u & =\partial_{\xi \xi} u+\partial_{\eta}\left(\left|\partial_{\eta} u\right|^{p-2} \partial_{\eta} u\right)=\partial_{\xi \xi} u+(p-1) u_{\eta}^{p-2} u_{\eta \eta} \\
& =|\nabla u| \Delta_{1} u+(p-1)|\nabla u|^{p-2} \Delta_{\infty} u
\end{aligned}
$$

i.e. a mean curvature operator (14) with a second order term corresponding to (3). Note that the second order term induce invariant smoothing of the image data. Since (16) is merely a special case of (3), we further relax the mean curvature term next to better reflect the trade-off between edgepreservation and obtained smoothness. Although this modification appears straight-forward, its implications are non-trivial, however.

The anisotropic $L_{(2, p)}$ operator is given by

Definition 2. The operator $\Delta_{(p, q)}$ is

$$
\Delta_{(p, q)} u=|\nabla u|^{q} \Delta_{1} u+(p-1)|\nabla u|^{p-2} \Delta_{\infty} u, \quad p \in[1,2], \quad q \geq 0,
$$

Remark 1. Definition 2 is a straight-forward relaxation of the exponents in (3), motivated by the discussion in above point 3. The introduction of $q$ in (17), defines an additional degree of freedom, allowing us to control the trade-off between $\Delta_{1}$ and $\Delta_{\infty}$, i.e., a trade-off between edge preservation and smoothness.

The corresponding evolution problem of the operator $\Delta_{(p, q)}$ is

Definition 3. The evolution problem of $\Delta_{(p, q)}$ is given by

$$
\left\{\begin{aligned}
\partial_{t} u-\Delta_{(p, q)} u & =0, \quad p \in[1,2], \quad q \geq 0 \\
u(0) & =u_{0} \\
\partial_{n} u & =0
\end{aligned}\right.
$$

We point out that $q=0, p=1$ yields the familiar isotropic TV regularizer and $q=1, p=2$ results in the heat equation, i.e., isotropic filtering. In the next section, we propose to couple $p$ and $q$ via a smooth non-linear transition function such that $\Delta_{(p, q)}$ can be thought of a spatially variant TV and isotropic regularizer.

\section{Variable coefficients}

\subsection{Coefficient coupling}

In the previous section we motivated the $\Delta_{(p, q)}$ operator and advocated to introduce variable coefficients, depending on the image data. The behavior of the operator $\Delta_{(p(x), q(x))}$ that we seek, is the edge-preserving effect of TV leading to $\Delta_{(p(x), q(x))} \rightarrow \Delta_{(1,0)}$ (the case of TV) as $|\nabla u| \rightarrow \infty$. In regions with small gradients we define the $(p(x), q(x))$-coefficients such that the operator is a linear filter. leading to $\Delta_{(p(x), q(x))} \rightarrow \Delta_{(2,1)}=\Delta$ (the case of isotropic diffusion) as $|\nabla u| \rightarrow 0$. To see how the diffusion appears in $\Delta_{(p(x), q(x))}$ we rewrite the operator in Gauge coordinates 
Lemma 1. The operator $\Delta_{(p, q)}$ in (17) can be written as

$$
\Delta_{(p, q)} u=|\nabla u|^{q-1}\left(\xi^{\top}\left(D^{2} u\right) \xi+(p-1)|\nabla u|^{p-q-1} \eta^{\top}\left(D^{2} u\right) \eta\right) .
$$

Proof. Using the relations in (6) and by rotation invariance of Laplacian, we obtain

$$
\Delta u=u_{\xi \xi}+u_{\eta \eta}=\Delta_{\infty} u+|\nabla u| \Delta_{1} u
$$

Now we observe that the operator $\Delta_{1} u$ can be expanded as

$$
\Delta_{1} u=\frac{u_{y}^{2} u_{x x}-2 u_{x} u_{y} u_{x y}+u_{x}^{2} u_{y y}}{|\nabla u|^{3}}=\frac{\left(\nabla^{\perp} u\right)^{\top}\left(D^{2} u\right) \nabla^{\perp} u}{|\nabla u|^{3}},
$$

and using this in (20) gives

$$
\Delta_{\infty} u=|\nabla u|^{2} \Delta u-\left(\nabla^{\perp} u\right)^{\top}\left(D^{2} u\right) \nabla^{\perp} u=\nabla^{\top} u\left(D^{2} u\right) \nabla u .
$$

Thus, the $\Delta_{(p, q)}$ operator in (17) reformulates to

$$
\begin{aligned}
\Delta_{(p, q)} u & =|\nabla u|^{q-3}\left(\nabla^{\perp} u\right)^{\top}\left(D^{2} u\right) \nabla^{\perp} u+(p-1)|\nabla u|^{p-4} \nabla^{\top} u\left(D^{2} u\right) \nabla u \\
& =|\nabla u|^{q-1} \xi^{\top}\left(D^{2} u\right) \xi+(p-1)|\nabla u|^{p-2} \eta^{\top}\left(D^{2} u\right) \eta,
\end{aligned}
$$

which shows the result.

Next, we couple $p$ and $q$ via the relation

$$
p(|\nabla u|)=1+q(|\nabla u|)
$$

and from which we derive the following result

Lemma 2. If $p(x)=1+q(x)$, then

$$
\Delta_{(1+q(x), q(x))} u=|\nabla u|^{q(x)-1}\left(\xi^{\top}\left(D^{2} u\right) \xi+q(x) \eta^{\top}\left(D^{2} u\right) \eta\right) .
$$

Proof. The proof follows immediately from Lemma 1.

In this study, we couple $p$ and $q$ via the negative exponential function (although other selections are possible), i.e., we set

$$
\begin{aligned}
& q(|\nabla u|)=k_{2} \exp \left(-|\nabla u| / k_{1}\right), \\
& p(|\nabla u|)=1+q(|\nabla u|),
\end{aligned}
$$

where $k_{1}>0$ and $0<k_{2}<1$. Under this selection we form the following parabolic PDE

$$
\partial_{t} u-|\nabla u|^{q(|\nabla u|)-1}\left(\xi^{\top}\left(D^{2} u\right) \xi+q(|\nabla u|) \eta^{\top}\left(D^{2} u\right) \eta\right)=0 .
$$

One easily checks that (26) describes a smooth transition between total variation and linear filtering for the selection of $p, q$ in (25). By using $|\nabla u| \Delta_{1} u=\Delta u-$ $\Delta_{\infty} u$, the operator $\Delta_{(1+q, q)}$ becomes

$$
\Delta_{(1+q, q)} u=|\nabla u|^{q(|\nabla u|)-1}\left(\operatorname{Tr}\left(D^{2} u\right)+\frac{q(|\nabla u|)-1}{|\nabla u|^{2}} \nabla u^{\top}\left(D^{2} u\right) \nabla u\right) .
$$


Remark 2. The operator $\Delta_{(1+q, q)}$ is non-linear and have unbounded coefficients. In this first study, we perturb the $\Delta_{(1+q, q)}$-operator to obtain a regularized version of the evolution problem (18). This regularization enables us pose the necessary conditions for well-posedness, introduced next.

\subsection{Regularity of solutions}

In order to set up the framework for numerical calculation, we define

Definition 4. Let $0<\varepsilon, \delta<1$ and $q$ as in (25). Then

$$
\begin{aligned}
& \Delta_{(1+q, q)}^{\varepsilon, \delta} u= \\
& \left(|\nabla u|^{2}+\varepsilon^{2}\right)^{(q(|\nabla u|)-1) / 2}\left((1+\delta) \operatorname{Tr}\left(D^{2} u\right)+\frac{q(|\nabla u|)-1}{|\nabla u|^{2}+\varepsilon^{2}} \nabla u^{\top}\left(D^{2} u\right) \nabla u\right) .
\end{aligned}
$$

Thus we study the regularized evolution equation

$$
u_{t}=\Delta_{(1+q, q)}^{\varepsilon, \delta} u=\sum_{i, j=1} a_{i j}^{\varepsilon, \delta}(\nabla u) u_{i j}
$$

where

$$
a_{i j}^{\varepsilon, \delta}(\zeta)=\left(|\zeta|^{2}+\varepsilon^{2}\right)^{(q(|\zeta|)-1) / 2}\left[(1+\delta) \delta_{i j}+\frac{q(|\zeta|)-1}{|\zeta|^{2}+\varepsilon^{2}} \zeta_{i} \zeta_{j}\right],
$$

and $\zeta=\nabla u$. Given $u^{(k)}$, we obtain the next update $u^{(k+1)}$ by solving the following, equivalent (see [16]), initial value problem iteratively

$$
\left\{\begin{aligned}
u_{t}^{(k+1)} & =\sum a_{i j}^{\varepsilon, \delta}\left(\nabla u^{(k)}\right) u_{i j}^{(k+1)} & & \text { in } \Omega \times(0, T) \\
u^{(k+1)}(0) & =u_{0}, & & \text { in } \Omega \\
\partial_{n} u^{(k+1)} & =0, & & \text { on } \partial \Omega \times(0, T)
\end{aligned}\right.
$$

Proposition 1. If the initial data $u^{(0)}=0$ then the solution $u^{(k)}$ to $(31)$ exists and is in $C^{\infty}(\Omega \times(0, T))$.

Proof. If the initial guess $u^{(0)}=0$, then $\sum a_{i j}^{\varepsilon, \delta}(0) u_{i j}=(1+\delta) \varepsilon^{q(0)-1} \Delta u$ and we deduce Thm 4.31 in [11] that $u^{(1)}$ exists and is $C^{\infty}(\Omega \times(0, T))$. Given $u^{(k)} \in C^{\infty}$, then $\left\|u^{(k)}\right\|_{C^{1}(\Omega \times(0, T))}$ is bounded, $\left(|\zeta|^{2}+\varepsilon^{2}\right)^{(q(|\zeta|)-1) / 2}$ is bounded from below and $a_{i j}^{\varepsilon, \delta}$ are also $C^{\infty}(\Omega \times(0, T))$. Hence, there are constants $c=c(\varepsilon, \delta, k)$ and $C=C(\varepsilon, \delta, k)>0$, depending only on $\varepsilon, \delta,\left\|u^{(k)}\right\|_{C^{1}}$, such that

$$
c|\zeta|^{2} \leq a_{i j}^{\varepsilon, \delta}\left(\nabla u^{(k)}\right) \zeta_{i} \zeta_{j} \leq C|\zeta|^{2} .
$$

It follows once again from Thm 4.31 in [11] that $u^{(k+1)}$ exists and also is $C^{\infty}(\Omega \times$ $(0, T))$. 


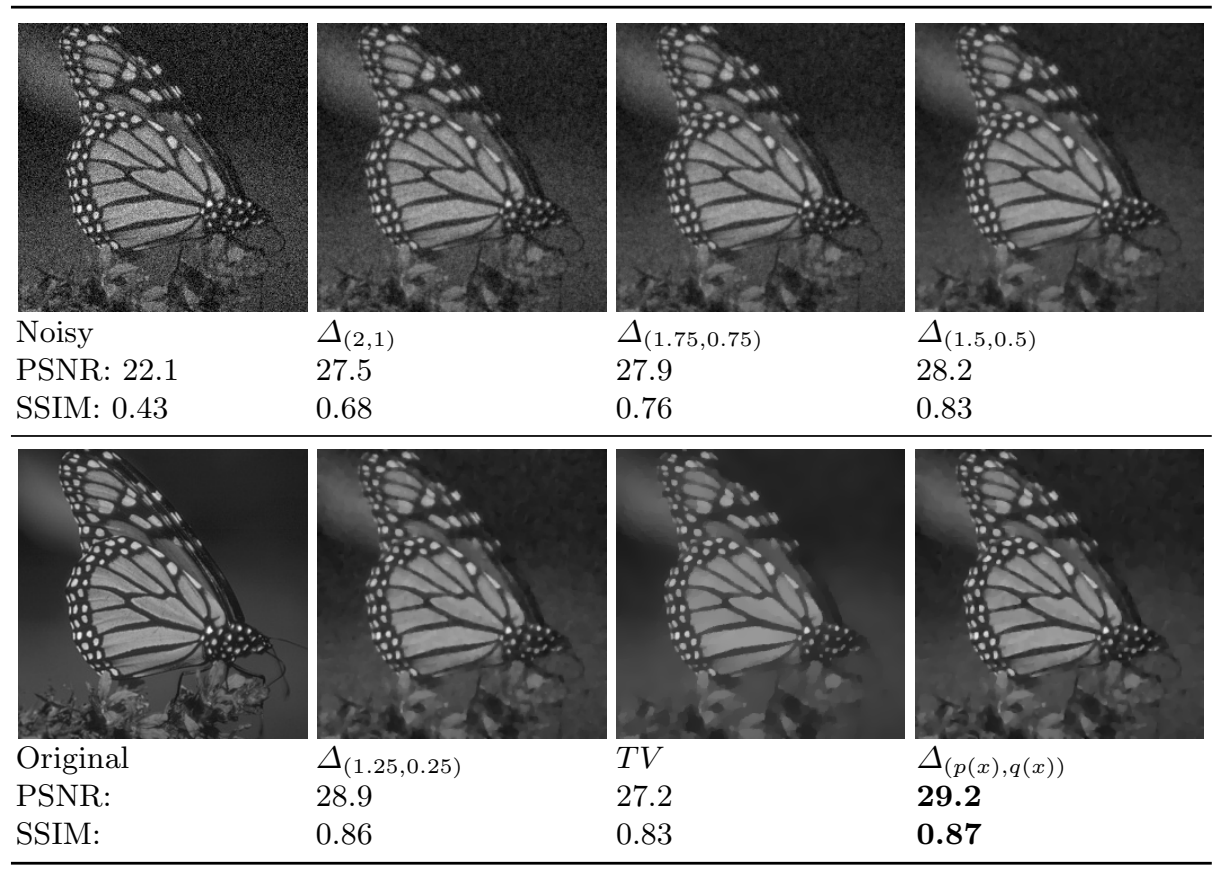

Fig. 2: Example results for the $\Delta_{(p, q)}$-operator where we stopped the filtering process at the maximum SSIM value. "TV"-was obtained using the Split Bregman method [9]. We set $k_{1}=k_{2}=0.1$ in $\Delta_{(p(x), q(x))}$. See text for details.

\section{Evaluation}

\subsection{Implementation}

The parabolic PDE is discretized by using finite differences and a simple forward Euler scheme. We used a state of the art Split Bregman (SB) implementation of total variation. For details on SB see [9]. The regularization parameter for the $\mathrm{SB}$ was optimized in the range $[0.01,1]$ in increasing steps of 0.11 . The regularization parameter of the Bregman-variables of $\mathrm{SB}$ was set to 1 and the scheme was terminated as $10^{-3}>\left\|u^{(k)}-u^{(k-1)}\right\|_{2} /\left\|u^{(k-1)}\right\|_{2}$. We choosed the regularization parameter that produces highest SSIM value [15]. The peak signalto-noise value (PSNR) is also reported. For the $(p(x), q(x))$-operator we found that $k_{1}=k_{2}=0.1$ and the update stepsize as $\alpha=10^{-5}$ and $\tau=0.5$ works well for the considered noise level of 20 standard deviations of additive Gaussian noise. These values are ad-hoc and future work include methods for parameter estimation.

\section{$5.2 \quad$ Results}

First we test our algorithm on a synthetic test image seen in figure 1 . The result of the proposed operator appears smoother than the result of TV in the center 


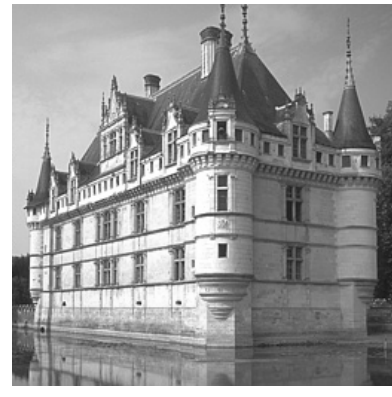

Original

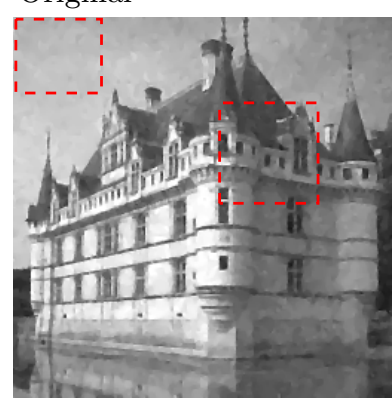

$\Delta_{(p(x), q(x))}$

PSNR (P): 25.9

$\operatorname{SSIM}(\mathrm{S}): \mathbf{0 . 8 1}$

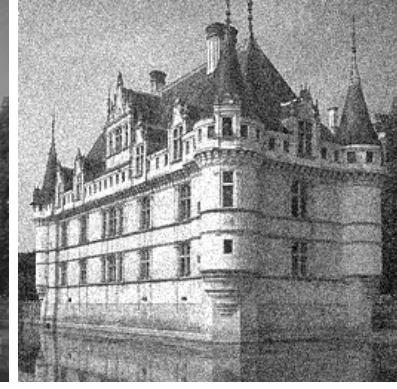

Noisy (P: 22.1, S: 0.54)

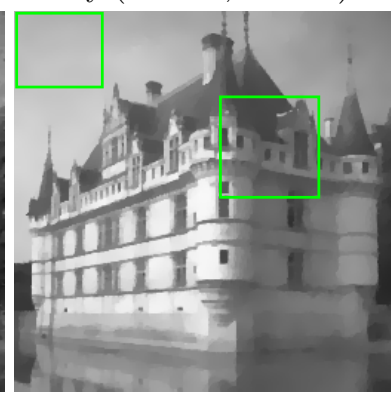

TV

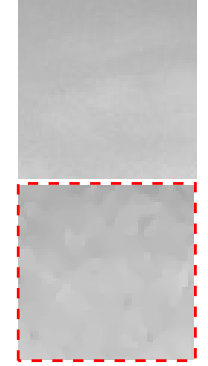

$\Delta_{(p(x), q(x))}$

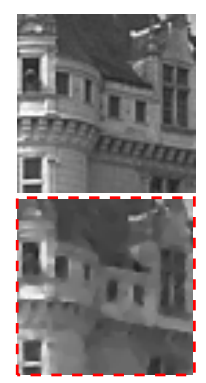

$\Delta_{(p(x), q(x))}$

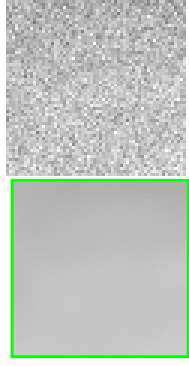

TV

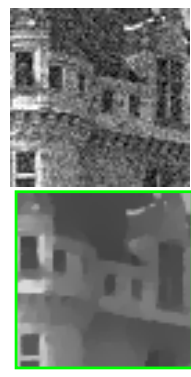

TV

Fig. 3: Example denoising a grayscale image with 20 standard deviations of noise. In the close-up images to the right it can be seen that TV (thick/green) produces the characteristic staircasing effect while the operator $\Delta_{(p, q)}($ dashed/red) shows good visual similarity with the noise free patch. TV shows good result in the sky (up right), but oversmooths, e.g., the window tiles seen in the close-up down right.

region, but yet preserves the corner point well. In figure 2 (cropped $256 \times 256$ pixels of image 35049.jpg [12]) the visual quality is compared and the SSIM-values for a range of $p, q$-values. As expected for $\Delta_{(2,1)}$ (isotropic filtering) performs the worst whereas the operator produce improved result w.r.t. SSIM as well as perceptual appearance. In the case of non-adaptive parameters, TV performs the best. However, the operator with adaptive coefficients improve both SSIM,PSNR values and produces less oversmoothing (down-right figure). The result from a grayscale image "Castle" (cropped $256 \times 256$ pixels of image 102061.jpg [12]) is included in figure 3. In the image TV performs very well in the sky (detail up right with green/thick frame) whereas the result from the $\Delta_{(p, q)}$ operator appears less noisy and looks visually more crisp. In both examples the proposed operator shows an improvement in PSNR as well as SSIM values. 


\section{Conclusion}

In this paper we introduced a new family of operators, $\Delta_{(p, q)}$. Preliminary numerical results indicate that there could be a relationship between $p(x)$ and $q(x)$ that further improves the restoration effect. In forthcoming works we will investigate the operator $\Delta_{(p, q)}$ further regarding both regularity and different areas of applications.

\section{References}

1. Alvarez, L., Lions, P.L., Morel, J.M.: Image selective smoothing and edge detection by nonlinear diffusion. SIAM J. Numer. Anal. 29(3), 845-866 (1992)

2. Antontsev, S.N., Shmarev, S.I.: Existence and uniqueness of solutions of degenerate parabolic equations with variable exponents of nonlinearity. Fundam. Prikl. Mat. 12(4), 3-19 (2006)

3. Antontsev, S., Shmarev, S.: Elliptic equations with anisotropic nonlinearity and nonstandard growth conditions. Handbook of differential equations. stationary partial differential equations. Amsterdam: Elsevier/North Holland 3, 1-100 (2006)

4. Aubert, G., Kornprobst, P.: Mathematical problems in image processing, Applied Mathematical Sciences, vol. 147. Springer, New York, second edn. (2006)

5. Baravdish, G., Svensson, O., Åström, F.: On Backward $p(x)$-Parabolic Equations for Image Enhancement. Numer. Funct. Anal. Optim. 36(2), 147-168 (2015)

6. Buades, A., Coll, B., Morel, J.M.: The staircasing effect in neighborhood filters and its solution. Image Processing, IEEE Trans. on 15(6), 1499-1505 (2006)

7. Chen, Y.G., Giga, Y., Goto, S.: Uniqueness and existence of viscosity solutions of generalized mean curvature flow equations. J. Diff Geom 33(3), 749-786 (1991)

8. Does, K.: An evolution equation involving the normalized $p$-laplacian. Commun. Pure Appl. Anal. 10(1), 361-396 (2011)

9. Goldstein, T., Osher, S.: The split bregman method for 11-regularized problems. SIAM Journal on Imaging Sciences 2(2), 323-343 (2009)

10. Keeling, S.L., Stollberger, R.: Nonlinear anisotropic diffusion filtering for multiscale edge enhancement. Inverse Problems 18(1), 175-190 (2002)

11. Lieberman, G.M.: Second order parabolic differential equations. World Scientific Publishing Co. Inc., River Edge, NJ (1996)

12. Martin, D., Fowlkes, C., Tal, D., Malik, J.: A database of human segmented natural images and its application to evaluating segmentation algorithms and measuring ecological statistics. In: ICCV. vol. 2, pp. 416-423 (2001)

13. Perona, P., Malik, J.: Scale-space and edge detection using anisotropic diffusion. IEEE Transactions, PAMI 12, 629-639 (1990)

14. Rudin, L.I., Osher, S., Fatemi, E.: Nonlinear total variation based noise removal algorithms. Physica D 60(1-4), 259-268 (1992)

15. Wang, Z., Bovik, A., Sheikh, H., Simoncelli, E.: Image quality assessment: from error visibility to structural similarity. Image Processing, IEEE Transactions on 13(4), 600-612 (2004)

16. Vogel and Oman: Iterative methods for total variation denoising, SIAM J. Sci. Comput. 17, pp.227-238, (1996)

17. Whitaker, R.T., Pizer, S.M.: A multi-scale approach to nonuniform diffusion. CVGIP: Image Understanding 57(1), 99-110 (1993)

18. You, Y.L., Xu, W., Tannenbaum, A., Kaveh, M.: Behavioral analysis of anisotropic diffusion in image processing. Image Processing, IEEE Trans. on 5(11), 1539-1553 (1996) 